\title{
LncRNA STK4 antisense RNA 1(STK4-AS1) promoted osteosarcoma by inhibiting p53 expression
}

\section{Weitao Yao}

Chuchu Wang ( $\nabla$ wangchuchu0108@163.com )

https://orcid.org/0000-0002-3936-4149

\section{Research Article}

Keywords: STK4-AS1, osteosarcoma, cell cycle, p53 protein

Posted Date: October 21st, 2021

DOI: https://doi.org/10.21203/rs.3.rs-1004881/v1

License: (c) (i) This work is licensed under a Creative Commons Attribution 4.0 International License.

Read Full License 


\section{Abstract}

Background: LncRNA STK4 antisense RNA 1(STK4-AS1) has been identified as a potential biomarker associated with multiple cancers. We proposed that STK4-AS1 plays a role in the proliferation of osteosarcoma by regulating the cell cycle.

Methods: We compared the expression of STK4-AS1, p53, and p21 in osteosarcoma vs normal samples in clinical tissues and cell lines. We determined the effect of overexpression and knockdown of STK4-AS1 in p53 expressing osteosarcoma cells U20S, p53 muted osteosarcoma cells MG63, and osteoblast cells hFOB on p53 and p21 expression and the cell viability. For U2OS and MG63, the cell cycle was analyzed and the expression of cyclin proteins was determined. We overexpressed p53 or p21 in STK4-AS1 overexpressed cells to explore the association of STK4-AS1 and p53 in U2OS.

Results: The STK4-AS1 expression was higher and p53 and p21 expression were lower in osteosarcoma tissue and cells than in their non-cancer counterparts. The expression of STK4-AS1 was negatively correlated with the expression of p53 or p21. Knockdown of STK4-AS1 in U2OS decreased the cell viability, increased cells in the G0/G1 phase, decreased cells in the $S$ and G2/M phase, decreased expression of cyclin A and B, increased p53 and p21, and had no effect on cyclin D and cyclin E, while overexpression of STK4-AS1 did the opposes. Overexpression of p53 or p21 recovered some changes caused by STK4-AS1 overexpression in U2OS. MG63 expressed no p53 and the expression of p21, cyclin $A$, and cyclin B, cell viability, and cell cycle were not affected by altered STK4-AS1 levels. In hFOB cells, the expression of p53 and p21 was decreased and the cell viability was increased when STK4-AS1 was overexpressed, but they were not affected when STK4-AS1 was knocked down.

Conclusion: LncRNA STK4-AS1 promoted the cell cycle of osteosarcoma cells by inhibiting p53 expression.

\section{Introduction}

Osteosarcoma is the most common primary bone malignancy [1]. The incidence of osteosarcoma is 5.4 cases per year per million in males and 4 cases per year per million in females [1]. Although the mortality for osteosarcoma has steadily declined by approximately $1.3 \%$ per year, the 5 -year overall survival rate is still lower than $70 \%$ [1]. Studies have revealed multiple regulation pathways of bone metabolism [2-6], and many effective therapeutic approaches to bone disease have been developed [7, 8]. Although many preclinical studies have contributed to clinical cancer treatment [9-12], to date, the understanding of the pathology of osteosarcoma is insufficient [13]. Hence, the study of osteosarcoma is necessary.

Long non-coding RNAs (IncRNAs) are RNAs whose lengths are over 200 bp but are not translated into protein [14]. LncRNAs function as transcriptional regulators for the expression of many genes [15]. Recently, more and more ncRNAs were found to be involved in bone cell regulations [16-19]. Many IncRNAs were identified to be associated with human diseases including cancers [20-22]. Studies revealed that the IncRNAs expressed in bone play a critical role in regulating bone formation [23] and 
differentiation [24-26]. Osteosarcoma derives from primitive bone-forming mesenchymal cells [1] and it is also suggested to be regulated by IncRNAs [27]. Recently popular cancer bioinformatics studies [28] showed that an increasing number of IncRNAs were identified as the prognostic biomarkers or potential therapeutic targets of cancer by bioinformatics study $[29,30]$ and the role of many of them in osteosarcoma has been validated in vivo or in vitro [31-34]. As the number of IncRNAs is large and the regulation network of IncRNAs is complex, more works are required in this field.

A IncRNA, STK4-AS1, has previously been identified as a cancer biomarker associated with the progression of breast cancer[35], lung cancer [30], and colon cancer [29]. STK4-AS1 orientates from the minus strand and is located at 20q13.12 in the human genome with a size of 2,665 bases [36]. It is one of the antisense RNAs of the STK4 gene. STK4 protein was reported to regulate many types of cancers, including liver cancer [37], prostate cancer [38], and thyroid carcinoma [39]. However, the effect of its antisense RNA was still unclear. Some IncRNAs can regulate cell growth by regulating the cell cycle [40]. We proposed that STK4-AS1 plays a role in the proliferation of osteosarcoma by regulating its cell cycle. In this study, we investigated the expression of IncRNA STK4-AS1 in osteosarcoma, both in clinical samples and cell models, and study its effect on the osteosarcoma cell cycle. Our study aims to deepen the understanding of the role of STK4-AS1 in osteosarcoma and develop it as a potential novel target for the treatment of osteosarcoma.

\section{Materials And Methods}

\subsection{Clinical tissue samples}

Clinical osteosarcoma and adjacent normal bone tissues were collected from osteosarcoma patients who underwent surgery from 2016 to 2019 in the Affiliated Tumor Hospital of Zhengzhou University (Hunan, China). This work was approved by the Ethical Committee of Zhengzhou University and all of the patients consented to participate in this study. Samples were immediately snap-frozen in liquid nitrogen for further study.

\subsection{Cell lines and cell culture}

Human osteosarcoma cell lines MG-63 (ATCC $\AA$ CRL-1427 ${ }^{\text {TM }}$ ), Saos-2 (ATCC $®$ HTB-85 $5^{\text {TM }}$ ), U-2 OS (ATCC $®$ HTB-96 ${ }^{\text {TM }}$ ), and human osteoblast cell line hFOB 1.19 (ATCC $®$ CRL-11372 ${ }^{\text {TM }}$ ) was obtained from ATCC (Washington, USA). Human primary osteoblast cell line HOB was obtained from PromoCell (Heidelberg, Germany). Cell lines were cultured in Osteoblast Growth Medium C-27001 (Heidelberg, Germany) in a humidified atmosphere of $5 \% \mathrm{CO}_{2}$ at $37^{\circ} \mathrm{C}$.

\subsection{MTT assay}

The MTT assay was first reported by Mossmann in 1983 [41]. It is commonly used for both cancer cell and non-cancer cell viability determination [42,43]. Briefly, cells were grown in 96 well plates and at the endpoint of the culture time, the culture medium was removed and $0.8 \mathrm{mg} / \mathrm{ml}$ of MTT working solution (dissolved in serum-free medium) was added to the wells. After 4 hours, DMSO was added to the wells, 
and absorbance was recorded at $560 \mathrm{~nm}$ using the microplate spectrophotometer system (Spectra max190-Molecular Devices).

\subsection{EdU assay}

The EdU assay was conducted using EdU Staining Proliferation Kit (iFluor 488) (ab219801) with BD FACSCalibur was used to acquire cell cycle data. FlowJo Version 10 was used to analyze the data. Briefly, EdU solution was added to cells and the cells were incubated for $4 \mathrm{hrs}$. Then cells were fixed and permeabilized. The cells were analyzed by flow cytometry.

\subsection{RNA extraction and QPCR}

The expression of STK4-AS1 was determined by QPCR assay which was described previously [44]. Total RNA from tissue or cell samples was extracted by using Trizol reagent (Invitrogen, Calsbad, CA, USA). Then the total RNA was reversely transcribed into cDNA using a reverse transcription kit (Thermo, USA). Quantitative real time-PCR was done with PowerUp ${ }^{\text {TM }}$ SYBR $^{\text {TM }}$ Green Master Mix (Thermo, USA) using the ViiA 7 Real-Time PCR System (Applied Biosystems). The protocol for PCR: $95^{\circ} \mathrm{C}$ for $3 \mathrm{~min}, 40$ cycles of $95^{\circ} \mathrm{C}$ for $30 \mathrm{~s}, 58^{\circ} \mathrm{C}$ for $15 \mathrm{~s}$, and $72^{\circ} \mathrm{C}$ for $30 \mathrm{~s}$. Gene expression was quantified using the $2^{-} \triangle \triangle \mathrm{CT}$ method. The primers used for RT-PCR were designed by Eurofine Genomic primer design online tools and synthesized by Sigma-Aldrich, Inc. (St. Louis, MO, USA). The sequences are as follows: GAPDH forward: 5'-AATGGGCAGCCGTTAGGAAA-3' and reverse: 5'-TGAAGGGGTCATTGATGGCA-3'. STK4-AS1 forward: 5'CGGAGCGCACAAAATACTCG-3' and reverse: 5'-CCACAAATACCTCCAGGCGT-3'.

\subsection{Cell transfection}

The STK4-AS1 knockdown and overexpression cells and p53 and p21 rescue cells were conducted by transfection of siRNA or expression vector. The cell lines were transfected using Lipofectamine ${ }^{\circledR} 2000$ (Invitrogen; Thermo Fisher Scientific, Inc.). The expression levels of STK4-AS1 were validated by qPCR. The expression of p53 and p21 was validated by western blotting. The cell transfection method was described previously [45].

The experimental details are as follows: (1) STK4-AS1 knockdown: STK4-AS1 small interfering (si)RNA and negative control siRNA were purchased from Sigma-Aldrich (St. Louis, MO, USA). The sequences were as follows: STK4-AS1 siRNA, 5'-

ACCTCGGCCAGAGAGGTGAAGTATCTTCAAGAGAGATACTTCACCTCTCTGGCCTT-3'. A scramble siRNA (siN05815122147) was used as the NC siRNA. The transfection concentration of siRNA was $60 \mathrm{nM}$. (2) STK4-AS1 overexpression: The entire sequence of human STK4-AS1 was amplified from hFOB cell lines using PCR and cloned into the pcDNA3.1 vector. The negative control empty vector, which was purchased from Sigma-Aldrich (St. Louis, MO, USA). The transfection concentration of the plasmid was $2 \mu \mathrm{g} / \mathrm{ml}$. (3) P53 or p21 rescue: p53 or p21 expressing vectors, TR200pa-ppGF-p53(or p21)-mCMV-EF1a-Puro (plasmid), and negative vectors were purchased from System Biosciences (Palo Alto, CA, USA). The transfection concentration of the plasmid was $2 \mu \mathrm{g} / \mathrm{ml}$. 
The control of cell transfection was set as follows: Ctrl1: cells without treatment; Ctrl2: cells with NC siRNA transfection; Ctrl3: cells with empty pcDNA3.1 vector transfection.

\subsection{Cell cycle analysis}

The cell cycle was analyzed using flow cytometry with propidium iodide (PI) staining, which was described previously [46]. Briefly, cells were washed with PBS and resuspended at a concentration of $1 \times$ $10^{6} / \mathrm{mL}$. Cells were fixed with $100 \%$ ethanol for three hours at $4^{\circ} \mathrm{C}$. Then suspended cells were washed with PBS two times and incubated with propidium iodide staining solution $(0.1 \%$ Triton $X-100,0.2 \mathrm{mg} / \mathrm{mL}$ DNAse-free RNAse A, $0.02 \mathrm{mg} / \mathrm{mL}$ in cold PBS) at $37^{\circ} \mathrm{C}$ for $15 \mathrm{~min}$. BD FACSCalibur was used to acquire cell cycle data. FlowJo Version 10 was used to analyze the data.

\subsection{Western blotting}

The western blotting method was described previously [47]. Briefly, proteins were extracted from cells using RIPA buffer with protease inhibitor (Sigma-Aldrich, USA). Total protein concentrations were determined using a BCA protein assay kit to control the loading amount $(25 \mu \mathrm{g})$. SDS gel electrophoresis was performed to separate proteins. Afterward, the proteins were transferred to $0.2-\mu \mathrm{m}$ polyvinylidene difluoride membranes which were subsequently blocked with $5 \%$ skim milk in Tris-buffered saline with $0.5 \%$ Tween- 20 (TBST). Membranes were then reacted with primary (1:1000 dilution of $5 \%$ skim milk in TBST) and secondary antibodies (1:3000 dilution of 5\% skim milk in TBST) subsequently. The band intensities were photographed using a super-sensitive gel imaging system after reacted with ECL reagents (Bio-Rad, Hercules, CA, USA).

The primary antibodies used in the experiment are as follow: Anti-cyclin A antibody (sc-271682), AntiCyclin B antibody (ab72), Anti-p53 antibody (ab26), Anti-p21 antibody (ab109520), Cyclin D1 Antibody (\#2922), and Anti-Cyclin E1 antibody (ab33911). The secondary antibodies are all purchased from Sigma-Aldrich (St. Louis, MO, USA).

\subsection{Interaction analysis of STK4-AS1 and TP53.}

All the analyses were conducted using LncRRIsearch [48] (http://rtools.cbrc.jp/LncRRIsearch/index.cgi).

\subsection{Statistics}

T-test or one-way ANOVA and Dunnett's post hoc tests were used to compare the significant difference between the control and experimental group $(p<0.01)$. GraphPad Prism (version 8$)$ was used to plot the figures and calculate statistical significance.

\section{Results}

\subsection{STK4-AS1 was overexpressed in osteosarcoma tissues}

This study started with clinical osteosarcoma samples. We collected clinical osteosarcoma and adjacent normal tissues from patients who underwent surgery and compared the STK4-AS1, p53, and p21 
expression in these samples. Results showed that STK4-AS1 expression in osteosarcoma samples was significantly higher than that in the adjacent normal tissues (Fig. 1A), suggesting that STK4-AS1 might be a cancer biomarker associated with the development of osteosarcoma. Results also showed that p53 and p21 expression was lower in osteosarcoma compared with the adjacent normal tissues both in mRNA and protein levels (Fig. 1B-F).

\subsection{STK4-AS1 was associated with p53/p21 regulation}

Based on these results, we suspected that STK4-AS1 might be associated with p53/p21 regulation, therefore, a correlation analysis between STK4-AS1 and p53 or p21 expression was conducted with these expressions data. Interestingly, both analyses revealed significant negative slopes with a p-value less than 0.05 (Fig. $1 \mathrm{GH}$ ), which strongly suggested an association between STK4-AS1 and p53/p21 regulation. We hypothesized that STK4-AS1 can inhibit the expression of p53 and p21.

\subsection{STK4-AS1 was overexpressed in osteosarcoma cells}

To further test this hypothesis, we also compared the expression of STK4-AS1 in osteosarcoma cells vs normal osteoblast cells. Results showed that the expression of STK4-AS1 in osteosarcoma cells U2OS, MG63, and SAOS-2 was much higher than in normal osteoblast cells hFOB and HOB (Fig. 2A). Although all three osteosarcoma cell lines tested expressed STK4-AS1, only U2OS expressed p53 while MG63 and SAOS-2 expressed almost no detectable level of p53 (Fig. 2B). According to previous studies, MG63 and SAOS- 2 cells have inactivated p53 while U2OS have wild-type p53 $[49,50]$. In addition, results showed that normal osteoblast cells hFOB and HOB expressed higher p53 and p21 compared to osteosarcoma cells (Fig. 2B-D).

\subsection{STK4-AS1 regulated p21 through p53.}

In order to get further insight into the effects of STK4-AS1 on osteosarcoma, we selected three typical cells with different p53 expression for the subsequent study, including p53 expressing osteosarcoma cells U2OS, p53 muted osteosarcoma cells MG63, and osteoblast cells hFOB. Cell transfection was used to knock down or overexpress STK4-AS1 in these three cell lines, which were validated by QPCR assay (Fig. 2E-G1). In p53 expressing U2Os, p53 was increased when STK4-AS1 was knocked down, while p53 was reduced when STK4-AS1 was overexpressed (Fig. 2F2-3). But in hFOB cells, p53 was not changed when STK4-AS1 was knocked down, while p53 was reduced when STK4-AS1 was overexpressed (Fig. 2G2-3). We suggested that in hFOB, STK4-AS1 was already low that unable to inhibit p53 expression, thus the knockdown did not eliminate the inhibition of p53, while the overexpression can suppress p53 expression. In U2O2 and hFOB cells, the alteration of p21 expression was similar to the changing trend of p51 (Fig. 2E2-3). This suggested that the STK4-AS1 inhibited p21 via reducing p53 signals. In addition, in p53 defected MG63 cells, the change of STK4-AS1 had no effect on p21 (Fig. 2G2-3), which also supported that p53 is critical for the regulation of STK4-AS1 toward p21 (Fig. 2E-G 2-3).

\subsection{STK4-AS1 regulated cell viability through p53}


As p53 and p21 are key regulators for cell viability, we determined the cell viability using MTT assay with our cell models. Results showed that knockdown of STK4-AS1 in osteosarcoma cells U2OS decreased cell proliferation while overexpression of STK4-AS1 increased cell proliferation (Fig. 2E4). Similar results were shown in the MTT assay of hFOB (Fig. 2G4). However, artificially altered levels of STK4-AS1 failed to affect the cell viability of p53 muted cell line MG63 (Fig. 2F4). This indicated that p53 might mediate the effects of STK4-AS1 on the viability of osteosarcoma and osteoblast cells. To confirm the effect of STK4-AS1 on cell viability and proliferation, we conducted the EdU assay. Results showed similar results as the MTT assay (Fig. 3). Therefore, we suggested that STK4-AS1 could regulate cell proliferation.

\subsection{Effect of STK4-AS1 on the cell cycle}

To further explore the exact effect of STK4-AS1 on cell growth, we measured its effect on the U2OS cell cycle. Results showed that, compared with the control, the knockdown of STK4-AS1 increased cells in G0/G1 phase and caused a significant decrease in the S phase and G2/M phase cells. On the other hand, the overexpression of STK4-AS1 decreased cells in G0/G1 phase but caused an increase in S phase cells and G2/M phase cells (Fig. $4 \mathrm{AB}$ ). This indicated that the expression of STK4-AS1 might facilitate cell division. Hence, we suggested that STK4-AS1 promotes osteosarcoma by regulating the cell cycle.

\subsection{Effect of STK4-AS1 on the expression of cyclin proteins}

This suggestion was confirmed by the determination of key regulators of the cell cycle, cyclin proteins. Western blotting experiments revealed that the knockdown of STK4-AS1 decreased the expression of cyclin A and cyclin B while the overexpression of STK4-AS1 did the opposite (Fig. 4 CD). Cyclin A is a critical factor to initiate and complete DNA replication [51,52] and Cyclin B regulates the $G 2$ checkpoint [53]. The alteration of these two critical factors further confirmed that STK4-AS1 promotes osteosarcoma by regulating the cell cycle. These results could account for the changes in cell numbers in the $S$ and $G 2$ phases. However, although cell in G1 phase was affected, G1 regulators cyclin D and cyclin E [54] were not affected (Fig. 4 CD).

\subsection{P53 mediated the regulation of STK4-AS1 on cell cycle}

Cancer suppressor p53 has long been reported to function as a cell cycle controlling protein in osteosarcomas [55]. It has been proved to regulate the $G 2$ phase by affecting cyclin B [56]. Here we tested it as a potential target underlying the effect of STK4-AS1 on osteosarcomas cells. Results showed that the knockdown of STK4-AS1 decreased the expressions of both p53 and p21 while the overexpression increased the expression of p53 and p21 (Fig. 5AB). Previously, the p53/p21 pathway was reported to regulate $\mathrm{G} 2 / \mathrm{M}$ cell cycle genes [57]. Our results provided evidence that p53 and p21 are potential downstream targets of STK4-AS1.

\subsection{Rescue experiments of $\mathrm{p} 53$ and $\mathrm{p} 21$ proteins in U2OS}

A rescue experiment of p53 and p21 in STK4-AS1 overexpressed U2OS cells was conducted to demonstrate the involvement of p53 and p21 in the effect of STK4-AS1 on U2OS cells. The western blotting validated that most of the p53 or p21 expression was successfully rescued in STK4-AS1 
overexpressed U2OS cells. Western blotting results showed that the rescue of p53 also upregulated the expressions of p21, cyclin A, and cyclin B, while the rescue of p21 did not increase p53 level, indicating that p21 was the downstream target of the STK4-AS1/p53 pathway. (Fig. 5CD). The recovery of p53 or p21 expressions also decreased the viability of STK4-AS1 overexpressed U2OS cells (Fig. 5E), indicating that p53 and p21 might mediate STK4-AS1 cell viability regulations. However, as p53 and p21 have many regulatory effects on cell viability, these results were not enough to confirm that p53/p21 was involved in U2OS cell viability regulations.

\subsection{STK4-AS1 did not affect p53 muted MG63 cells}

To confirm that p53 is essential for STK4-AS1 cell cycle regulations, we repeated the cell cycle analysis in MG63 cells. As MG63 cells naturally mute the p53 expression (As we confirmed in western blotting, Fig. 2F2-3), it can be a proper negative control for our hypothesis. Results showed that both knockdown and overexpression of STK4-AS1 failed to make any difference in the cell cycle of MG63 cells (Fig. 6 AB) and the expression of cyclin A and cyclin B (Fig. 6 CD). These results supported that p53 is essential for STK4-AS1 cell cycle regulations.

\subsection{STK4-AS1 regulated cell cycle in osteoblast cells hFOB}

We have previously shown that STK4-AS1 overexpression in osteoblast cells hFOB brings down p53 and p21 levels while promoting proliferation. To explore whether p53/p21 mechanism was also presented in the cell cycle of hFOB cells, we repeated the cell cycle analysis in hFOB cells. Results showed that the knockdown of STK4-AS1 failed to alter the cell cycle and cell cyclinA and cyclinB. However, the overexpression of STK4-AS1 resulted in a decrease in G0/G1 and an increase in S/G2/M. In addition, the overexpression of STK4-AS1 also promoted the expression of cyclin A and cyclinB (Fig. 7). These results supported that upregulation of STK4-AS1 in normal osteoblasts would mimic malignant phenotype.

\subsection{Rescue experiments of p53 and p21 proteins in hFOB}

To further confirm that the p53/p21 mechanism was involved in the cell proliferation, a rescue experiment of $p 53$ and p21 in STK4-AS1 overexpressed hFOB cells was conducted. The western blotting validated that p53 and p21 expression was successfully rescued in STK4-AS1 overexpressed hFOB cells (Fig. 8AB). Western blotting results showed that the rescue of p53 also upregulated the expressions of p21, cyclin $A$, and cyclin B, while the rescue of p21 did not increase p53 level, which is consistent with the results in U2OS (Fig. 8CD). The rescue of p53 or p21 or p53/p21 co-rescue also decreased the viability of STK4AS1 overexpressed hFOB cells (Fig. 8E), indicating that p53 and p21 might also mediate STK4-AS1 cell viability regulations in $\mathrm{hFOB}$ cells.

\subsection{Interaction of STK4-AS1 and TP53.}


To explore whether STK4-AS1 directly interacted with TP53, we analyzed the subcellular localization (subcytoplasm and subnucleus compartments) of STK4-AS1 and TP53 and predicted the potential basepairing between STK4-AS1 and TP53 mRNA. Results showed that STK4-AS1 was mostly presented at membrane and cytosolsub, and it was also found at insoluble, nucleolus, nucleoplasm, and nucleussub. Results also suggested that STK4-AS1 might have sufficient assessable of TP53 mRNA at these subcellular localizations. The base-pairing interaction analysis revealed two interactions between STK4AS1 and TP53 mRNA. The total local base-pairing interaction energy between STK4-AS1 and TP53 mRNA was $-49.17 \mathrm{kcal} / \mathrm{mol}$ (Fig. 9). Therefore, we believed STK4-AS1 can directedly interact with the TP53 mRNA, thereby regulating TP53 protein.

\section{Discussion}

This study revealed the role of one IncRNA STK4-AS1 in the proliferation of osteosarcoma. From clinical samples, we found an association between the expression of STK4-AS1 and osteosarcoma: the cancer bone tissue expressed a higher level of STK4-AS1 over noncancer bone tissues. Thus, STK4-AS1 can be developed as a potential clinical prognostic biomarker for osteosarcoma. We also found that the expression of STK4-AS1 in osteosarcoma is correlated with p53 and p21 expression in tissue samples. As $p 53$ and p21 are critical regulators for cancers, this study revealed that STK4-AS1 can be a potential pharmacological target for osteosarcoma treatment. In addition, we suggested that STK4-AS1 might have different expression profiles in a certain fraction of cells within one sample. For example, cancer stem cells [58] might express a different level of STK4-AS1 from the other cancer cells.

Among the three osteosarcoma cell lines tested in this study, MG63 expressed most STK4-AS1. However, MG63 had no p53 expression, thus, it is a good negative control cell line for us to explore the role of p53 in STK4-AS1 regulation. We found that the actions of STK4-AS1 are different in U2OS and MG63. In U2OS, STK4-AS1 regulated the cell cycle while in MG63 STK4-AS1 showed not regulatory effects on cell viability or cell cycle. We suggested that the difference od p53 expression in U2OS and MG63 accounts for these differences. We also discovered that the viability of osteoblast cells $\mathrm{fHOB}$ only altered by overexpression of STK4-AS1 but not the knockdown. We suggested that this is because STK4-AS1 expression in $\mathrm{fHOB}$ is too low to regulate cell viability.

STK4-AS1 was found to be the "booster" in breast cancer [35], lung cancer[30], and colon cancer[29]. In this study, we demonstrated that it promoted the progression of osteosarcoma by inhibiting the p53/p21 pathway. MTT assay with transfected cells revealed that the expression of STK4-AS1 level was associated with cell viability. Many pharmacological targets were discovered because they can regulate cell viability $[59,60]$. We analyzed the effect of STK4-AS1 on the cell cycle of the p53 expressing osteosarcoma cell line U2OS and found that STK4-AS1 affected cell viability by regulating the cell cycle. Although some studies showed that the arresting of the cell cycle at the S or G2 phase can also lead to suppression of proliferation [61,62], based on our viability results, we suggested that the increased cells in S and G2 phase resulted in more proliferation of U2OS cells. 
In western blotting experiments, we observed the effect of STK4-AS1 on cyclin A, a regulator of the S phase regulation [63], cyclin $B$, a regulator of the $G 2$ phase [53], and cyclin D/cyclin $E$, regulators of the $G 1$ phase [54]. Furthermore, we also determined p53/p21 pathway. P53 has long been reported to regulate the cell cycle in osteosarcomas [55]. Here we tested it as a potential target underlying the effect of STK4AS1 on osteosarcomas cells. The p53/p21 pathway is responsible for the negative regulation of cyclin B and cdk1, regulating activities of the $G 2$ phase in cancer cells[53]. The accumulation of p53 can increase the expression of its transcriptional target gene $\mathrm{p} 21$ which can potentially inactivate the cyclin $\mathrm{B} / \mathrm{cdk} 1$ complex [64]. Our results showed that the knockdown or overexpression of STK4-AS1 affects the expression of p53, and the p53 subsequently affected p21 and negatively regulates activities in the $G 2$ phase. P53 rescue experiment showed that the abnormal cell viability, p21 expression, cyclin A expression, and cyclin B expression were largely recovered. Besides, p21 can also potentially binds and inactivate the cyclin E/CDK2 complex. Interestingly, although cyclin D and cyclin E have been found regulated by the $p 53 / p 21$ pathway $[65,66]$, in this study the cyclin $D$ and cyclin E levels were not affected even with p53/p21 level altered. We suggested that STK4-AS1 might have other impacts on cyclin D and cyclin $E$ besides the p53/p21 pathway that subset the effect of the p53/p21 pathway (Fig. 10). We supposed that this unidentified regulation might involve other cancer-related mechanisms such as ion channel regulations $[67,68]$. On the other hand, in the p53 negative control, MG63 cells, STK-AS1 failed to affect cyclin $A$ and cyclin $B$, which was consistent with our hypothesis.

As STK4 has known roles in regulating cancer progression, it might be helpful to distinguish the impact of STK4-AS1 from the potential effects of STK4 upregulation. Although STK4-AS1 is transcribed from the non-coding region of the STK4 gene, we determined the levels of STK4 protein in tumor tissues and cell lines, as well as in cell lines involving knockdown and overexpression of STK4-AS1. Results showed that there is no correlation between STK4-AS1 and STK4, and the expression of STK4 was not affected by the alteration of STK4-AS1 expression in the knockdown and overexpression experiments (SFig.1). In addition, we suggested that the study might greatly benefit from the data on patient prognosis. Thus, we analyzed the available survival data in this study (SFig.2). We were also interested in the effect of STK4AS1 on therapy response. However, the case number in this study was low and patients were treated with different therapy, including traditional medicine that was complex in their components, hence, we did not have enough cases to conclude the association of STK4-AS1 levels and patient survival or therapy response.

This study demonstrated that IncRNA STK4-AS1 is overexpressed in osteosarcoma and plays a role in the proliferation of p53 expressing osteosarcoma cells by affecting the cell cycle, which is mediated by the p53/p21 pathway. Given the potential value of STK4-AS1 for clinical osteosarcoma therapy and diagnosis, our study is conducive to the development of STK4-AS1 as a novel treatment target and diagnostic biomarker for osteosarcoma. In future clinical settings, STK4-AS1 can be determined using PCR or tested as one of the screening items in microarray probe assays. Potential gene therapy to reduce STK4-AS1 is also promising. 


\section{Declarations}

\section{Ethics approval and consent to participate}

This work was approved by the Ethical Committee of Affiliated Tumor Hospital of Zhengzhou University.

\section{Availability of data and materials}

All data generated or analyzed during this study are included in this published article and the supplementary materials.

\section{Competing interests}

There is no conflict of interest in this paper.

\section{Funding}

This work was supported by grants from the Affiliated Tumor Hospital of Zhengzhou University, China.

\section{Authors' contributions}

CONCEPTION: Chuchu Wang; INTERPRETATION OR ANALYSIS OF DATA: Weitao Yao and Jingyu Hou; PREPARATION OF THE MANUSCRIPT: Jingyu Hou, Guoqing Liu, Fangxing Wu, and Qiang Yan; REVISION FOR IMPORTANT INTELLECTUAL CONTENT: Liangyu Guo and Chuchu Wang; SUPERVISION: Liangyu Guo and Chuchu Wang.

\section{Acknowledgments}

We thank the support of Zhengzhou University.

\section{Abbreviations}

STK4-AS1

LncRNA STK4 antisense RNA 1

IncRNAs

Long non-coding RNAs

\section{References}

1. G. Ottaviani and N. Jaffe, The epidemiology of osteosarcoma, in: Pediatric and adolescent osteosarcoma, Springer, 2009, pp. 3-13.

2. G. Chen, C. Wang, J. Wang, S. Yin, H. Gao, L.U. Xiang, H. Liu, Y. Xiong, P. Wang, X. Zhu, L.I. Yang and R. Zhang, Antiosteoporotic effect of icariin in ovariectomized rats is mediated via the Wnt/betacatenin pathway, Exp Ther Med 12 (2016), 279-287. 
3. H. Liu, Y. Xiong, H. Wang, L. Yang, C. Wang, X. Liu, Z. Wu, X. Li, L. Ou, R. Zhang and X. Zhu, Effects of water extract from epimedium on neuropeptide signaling in an ovariectomized osteoporosis rat model, J Ethnopharmacol 221 (2018), 126-136.

4. H. Liu, Y. Xiong, X. Zhu, H. Gao, S. Yin, J. Wang, G. Chen, C. Wang, L. Xiang, P. Wang, J. Fang, R. Zhang and L. Yang, Icariin improves osteoporosis, inhibits the expression of PPARgamma, C/EBPalpha, FABP4 mRNA, N1ICD and jagged1 proteins, and increases Notch2 mRNA in ovariectomized rats, Exp Ther Med 13 (2017), 1360-1368.

5. X. Liu, H. Liu, Y. Xiong, L. Yang, C. Wang, R. Zhang and X. Zhu, Postmenopausal osteoporosis is associated with the regulation of SP, CGRP, VIP, and NPY, Biomed Pharmacother 104 (2018), 742750 .

6. Z. Wu, L. Ou, C. Wang, L. Yang, P. Wang, H. Liu, Y. Xiong, K. Sun, R. Zhang and X. Zhu, Icaritin induces MC3T3-E1 subclone14 cell differentiation through estrogen receptor-mediated ERK1/2 and p38 signaling activation, Biomed Pharmacother 94 (2017), 1-9.

7. W. Haixia, M. Shu, Y. Li, W. Panpan, S. Kehuan, X. Yingquan, L. Hengrui, L. Xiaoguang, W. Zhidi and O. Ling, Effectiveness associated with different therapies for senile osteopo-rosis: a network Metaanalysis, J Tradit Chin Med 40 (2020), 17-27.

8. C. Wang, G. Chen, J. Wang, H. Liu, Y. Xiong, P. Wang, L. Yang, X. Zhu and R. Zhang, Effect of Herba Epimedium Extract on Bone Mineral Density and Microstructure in Ovariectomised Rat, Journal of Pharmaceutical and Biomedical Sciences 6 (2016).

9. H. Liu, Effect of Traditional Medicine on Clinical Cancer, Biomedical Journal of Scientific \& Technical Research 30 (2020), 23548-23551.

10. H. Liu, A clinical mini-review: Clinical use of Local anesthetics in cancer surgeries, The Gazette of Medical Sciences 1 (2020), 030-034.

11. R. Li, H. Liu, J.P. Dilger and J. Lin, Effect of Propofol on breast Cancer cell, the immune system, and patient outcome, BMC Anesthesiol 18 (2018), 77.

12. R. Li, Y. Huang, H. Liu, J.P. Dilger and J. Lin, Comparing volatile and intravenous anesthetics in a mouse model of breast cancer metastasis, American Association for Cancer Research, 2018, pp. 2162.

13. S. Prater and B. McKeon, Cancer, Osteosarcoma, in: StatPearls, StatPearls Publishing Copyright (C) 2020, StatPearls Publishing LLC., Treasure Island (FL), 2020.

14. D. Meseure, K. Drak Alsibai, A. Nicolas, I. Bieche and A. Morillon, Long Noncoding RNAs as New Architects in Cancer Epigenetics, Prognostic Biomarkers, and Potential Therapeutic Targets, Biomed Res Int 2015 (2015), 320214.

15. L. Ma, J. Cao, L. Liu, Q. Du, Z. Li, D. Zou, V.B. Bajic and Z. Zhang, LncBook: a curated knowledgebase of human long non-coding RNAs, Nucleic Acids Research 47 (2019), D128.

16. F. Ou, K. Su, J. Sun, W. Liao, Y. Yao, Y. Zheng and Z. Zhang, The LncRNA ZBED3-AS1 induces chondrogenesis of human synovial fluid mesenchymal stem cells, Biochemical \& Biophysical Research Communications 487 (2017), 457-463. 
17. W. Zhuang, X. Ge, S. Yang, M. Huang, W. Zhuang, P. Chen, X. Zhang, J. Fu, J. Qu and B. Li, Upregulation of IncRNA MEG3 Promotes Osteogenic Differentiation of Mesenchymal Stem Cells from Multiple Myeloma Patients by Targeting BMP4 Transcription, Stem Cel/s 33 (2015), 19851997.

18. M. Mulati, Y. Kobayashi, A. Takahashi, H. Numata, M. Saito, Y. Hiraoka, H. Ochi, S. Sato, Y. Ezura, M. Yuasa, T. Hirai, T. Yoshii, A. Okawa and H. Inose, The long noncoding RNA Crnde regulates osteoblast proliferation through the Wnt/ $\beta$-catenin signaling pathway in mice, Bone 130 (2020), 115076.

19. X. Li, B. Peng, X. Zhu, P. Wang, Y. Xiong, H. Liu, K. Sun, H. Wang, L. Ou, Z. Wu, X. Liu, H. He, S. Mo, X. Peng, Y. Tian, R. Zhang and L. Yang, Changes in related circular RNAs following ERbeta knockdown and the relationship to rBMSC osteogenesis, Biochem Biophys Res Commun 493 (2017), 100-107.

20. W.J. Lukiw, P. Handley, L. Wong and D.R. Crapper McLachlan, BC200 RNA in normal human neocortex, non-Alzheimer dementia (NAD), and senile dementia of the Alzheimer type (AD), Neurochemical Research 17 (1992), 591-597.

21. X. Fu, L. Ravindranath, N. Tran, G. Petrovics and S. Srivastava, Regulation of apoptosis by a prostatespecific and prostate cancer-associated noncoding gene, PCGEM1, Dna \& Cell Biology 25 (2006), $135-41$.

22. J.B. Watson and J.G. Sutcliffe, Primate brain-specific cytoplasmic transcript of the Alu repeat family, Molecular \& Cellular Biology 7 (1987), 3324-3327.

23. J. Wang, S. Liu, J. Shi, H. Liu, J. Li, S. Zhao and Z. Yi, The Role of IncRNAs in Osteogenic Differentiation of Bone Marrow Mesenchymal Stem Cells, Curr Stem Cell Res Ther 15 (2020), 243249.

24. Y. Huang, Y. Zheng, L. Jia and W. Li, Long Noncoding RNA H19 Promotes Osteoblast Differentiation Via TGF-ß1/Smad3/HDAC Signaling Pathway by Deriving miR-675, Stem Cells 33 (2015), $3481-92$.

25. Q. He, S. Yang, X. Gu, M. Li, C. Wang and F. Wei, Long noncoding RNA TUG1 facilitates osteogenic differentiation of periodontal ligament stem cells via interacting with Lin28A, Cell Death Dis 9 (2018), 455.

26. Y. Gao, F. Xiao, C. Wang, C. Wang, P. Cui, X. Zhang and X. Chen, Long noncoding RNA MALAT1 promotes osterix expression to regulate osteogenic differentiation by targeting miRNA-143 in human bone marrow-derived mesenchymal stem cells, J Cell Biochem 119 (2018), 6986-6996.

27. S. Ghafouri-Fard, Z. Shirvani-Farsani, B.M. Hussen and M. Taheri, The critical roles of IncRNAs in the development of osteosarcoma, Biomed Pharmacother 135 (2021), 111217.

28. Y. Li, H. Liu and Y. Han, Potential Roles of Cornichon Family AMPA Receptor Auxiliary Protein 4 (CNIH4) in Head and Neck Squamous Cell Carcinoma, (2021).

29. F. Chen, Z. Li, C. Deng and H. Yan, Integration analysis for novel IncRNA markers predicting tumor recurrence in human colon adenocarcinoma, Journal of translational medicine 17 (2019), 299.

30. F. Qiao, N. Li and W. Li, Integrative bioinformatics analysis reveals potential long non-coding RNA biomarkers and analysis of function in non-smoking females with lung cancer, Medical science monitor: international medical journal of experimental and clinical research 24 (2018), 5771. 
31. R.M. Zhang, T. Tang, H.M. Yu and X.D. Yao, LncRNA DLX6-AS1/miR-129-5p/DLK1 axis aggravates stemness of osteosarcoma through Wnt signaling, Biochem Biophys Res Commun 507 (2018), 260266.

32. D. Zhao, S. Wang, X. Chu and D. Han, LncRNA HIF2PUT inhibited osteosarcoma stem cells proliferation, migration and invasion by regulating HIF2 expression, Artif Cells Nanomed Biotechnol 47 (2019), 1342-1348.

33. G. Kong, X.J. Qi and J.F. Wang, Effect of IncRNA LET on proliferation and invasion of osteosarcoma cells, Eur Rev Med Pharmacol Sci 22 (2018), 1609-1614.

34. D. Fu, C. Lu, X. Qu, P. Li, K. Chen, L. Shan and X. Zhu, LncRNA TTN-AS1 regulates osteosarcoma cell apoptosis and drug resistance via the miR-134-5p/MBTD1 axis, Aging (Albany NY) 11 (2019), 83748385.

35. L. Zhong, G. Lou, X. Zhou, Y. Qin, L. Liu and W. Jiang, A six-long non-coding RNAs signature as a potential prognostic marker for survival prediction of ER-positive breast cancer patients, Oncotarget 8 (2017), 67861.

36. Finishing the euchromatic sequence of the human genome, Nature 431 (2004), 931-45.

37. W. Li, J. Xiao, X. Zhou, M. Xu, C. Hu, X. Xu, Y. Lu, C. Liu, S. Xue, L. Nie, H. Zhang, Z. Li, Y. Zhang, F. Ji, L. Hui, W. Tao, B. Wei and $\mathrm{H}$. Wang, STK4 regulates TLR pathways and protects against chronic inflammation-related hepatocellular carcinoma, J Clin Invest 125 (2015), 4239-54.

38. D. Ready, K. Yagiz, P. Amin, Y. Yildiz, V. Funari, S. Bozdag and B. Cinar, Mapping the STK4/Hippo signaling network in prostate cancer cell, PLoS One 12 (2017), e0184590.

39. L.X. Yang, J. Wu, M.L. Guo, Y. Zhang and S.G. Ma, Suppression of long non-coding RNA TNRC6C-AS1 protects against thyroid carcinoma through DNA demethylation of STK4 via the Hippo signalling pathway, Cell Prolif 52 (2019), e12564.

40. Q. Sun, V. Tripathi, J.H. Yoon, D.K. Singh, Q. Hao, K.W. Min, S. Davila, R.W. Zealy, X.L. Li, M. Polycarpou-Schwarz, E. Lehrmann, Y. Zhang, K.G. Becker, S.M. Freier, Y. Zhu, S. Diederichs, S.G. Prasanth, A. Lal, M. Gorospe and K.V. Prasanth, MIR100 host gene-encoded IncRNAs regulate cell cycle by modulating the interaction between HuR and its target mRNAs, Nucleic Acids Res 46 (2018), 10405-10416.

41. T. Mosmann, Rapid colorimetric assay for cellular growth and survival: application to proliferation and cytotoxicity assays, J Immunol Methods 65 (1983), 55-63.

42. G. Fotakis and J.A. Timbrell, In vitro cytotoxicity assays: comparison of LDH, neutral red, MTT and protein assay in hepatoma cell lines following exposure to cadmium chloride, Toxicol Lett 160 (2006), 171-7.

43. H. Liu, J.P. Dilger and J. Lin, Effects of local anesthetics on cancer cells, Pharmacology \& Therapeutics 212 (2020), 107558.

44. B. Thornton and C. Basu, Rapid and simple method of qPCR primer design, Methods Mol Biol 1275 (2015), 173-9. 
45. L. Cervera, S. Gutiérrez-Granados, M. Martínez, J. Blanco, F. Gòdia and M.M. Segura, Generation of HIV-1 Gag VLPs by transient transfection of HEK 293 suspension cell cultures using an optimized animal-derived component free medium, J Biotechnol 166 (2013), 152-65.

46. P. Pozarowski and Z. Darzynkiewicz, Analysis of cell cycle by flow cytometry, Methods Mol Biol 281 (2004), 301-11.

47. M. Shen, Y. Feng, C. Gao, D. Tao, J. Hu, E. Reed, Q.Q. Li and J. Gong, Detection of cyclin b1 expression in $\mathrm{g}(1)$-phase cancer cell lines and cancer tissues by postsorting Western blot analysis, Cancer Res 64 (2004), 1607-10.

48. T. Fukunaga, J. Iwakiri, Y. Ono and M. Hamada, LncRRIsearch: A Web Server for IncRNA-RNA Interaction Prediction Integrated With Tissue-Specific Expression and Subcellular Localization Data, Front Genet 10 (2019), 462.

49. L. Ottaviano, K.L. Schaefer, M. Gajewski, W. Huckenbeck, S. Baldus, U. Rogel, C. Mackintosh, E. de Alava, O. Myklebost, S.H. Kresse, L.A. Meza-Zepeda, M. Serra, A.M. Cleton-Jansen, P.C. Hogendoorn, H. Buerger, T. Aigner, H.E. Gabbert and C. Poremba, Molecular characterization of commonly used cell lines for bone tumor research: a trans-European EuroBoNet effort, Genes Chromosomes Cancer 49 (2010), 40-51.

50. M. Roepke, A. Diestel, K. Bajbouj, D. Walluscheck, P. Schonfeld, A. Roessner, R. Schneider-Stock and H. Gali-Muhtasib, Lack of p53 augments thymoquinone-induced apoptosis and caspase activation in human osteosarcoma cells, Cancer Biol Ther 6 (2007), 160-9.

51. N. Bendris, B. Lemmers, J.M. Blanchard and N. Arsic, Cyclin A2 mutagenesis analysis: a new insight into CDK activation and cellular localization requirements, PLoS One 6 (2011), e22879.

52. M. Pagano, R. Pepperkok, F. Verde, W. Ansorge and G. Draetta, Cyclin A is required at two points in the human cell cycle, Embo j 11 (1992), 961-71.

53. Y. Ma, Y. Chen, C. Lin and G. Hu, Biological functions and clinical significance of the newly identified long non-coding RNA RP1-85F18.6 in colorectal cancer, Oncology Reports.

54. S.M. Keenan, N.H. Lents and J.J. Baldassare, Expression of cyclin E renders cyclin D-CDK4 dispensable for inactivation of the retinoblastoma tumor suppressor protein, activation of E2F, and G1-S phase progression, J Biol Chem 279 (2004), 5387-96.

55. L. Diller, J. Kassel, C.E. Nelson, M.A. Gryka, G. Litwak, M. Gebhardt, B. Bressac, M. Ozturk, S.J. Baker and B. Vogelstein, p53 functions as a cell cycle control protein in osteosarcomas, Molecular and cellular biology 10 (1990), 5772-5781.

56. S.A. Innocente, J.L. Abrahamson, J.P. Cogswell and J.M. Lee, p53 regulates a G2 checkpoint through cyclin B1, Proc Natl Acad Sci U S A 96 (1999), 2147-52.

57. M. Fischer, M. Quaas, L. Steiner and K. Engeland, The p53-p21-DREAM-CDE/CHR pathway regulates G2/M cell cycle genes, Nucleic Acids Res 44 (2016), 164-74.

58. H. Liu, A Prospective for the Potential Effect of Local Anesthetics on Stem-Like Cells in Colon Cancer, Biomedical Journal of Scientific \& Technical Research 25 (2020), 18927-18930. 
59. R. Li, C. Xiao, H. Liu, Y. Huang, J.P. Dilger and J. Lin, Effects of local anesthetics on breast cancer cell viability and migration, BMC cancer 18 (2018), 666.

60. H. Liu, J.P. Dilger and J. Lin, Lidocaine Suppresses Viability and Migration of Human Breast Cancer Cells: TRPM7 as A Target for Some Breast Cancer Cell Lines, Cancers (Basel) 13 (2021), 234.

61. H. Wang, Y. Chen, N. Zhai, X. Chen, F. Gan, H. Li and K. Huang, Ochratoxin A-Induced Apoptosis of IPEC-J2 Cells through ROS-Mediated Mitochondrial Permeability Transition Pore Opening Pathway, $J$ Agric Food Chem 65 (2017), acs.jafc.7b04434.

62. H. Liu, J.P. Dilger and J. Lin, The Role of Transient Receptor Potential Melastatin 7 (TRPM7) in Cell Viability: A Potential Target to Suppress Breast Cancer Cell Cycle, Cancers (Basel) 12 (2020).

63. H. Ying and Z.-X.J. Xiao, Targeting Retinoblastoma Protein for Degradation by Proteasomes, Cell Cycle 5 (2006), 506-508.

64. J.C. Marine and G. Lozano, Mdm2-mediated ubiquitylation: p53 and beyond, Cell Death \& Differentiation 17 (2010), 93-102.

65. T. Soucek, O. Pusch, E. Hengstschläger-Ottnad, P.D. Adams and M. Hengstschläger, Deregulated expression of E2F-1 induces cyclin A- and E-associated kinase activities independently from cell cycle position, Oncogene 14 (1997), 2251-7.

66. A.J. Levine, p53, the cellular gatekeeper for growth and division, Cell 88 (1997), 323-31.

67. H. Liu, Nav channels in cancers: Nonclassical roles, Global Journal of Cancer Therapy 6 (2020), 5.

68. H. Liu, A prospective for the role of two-pore channels in breast cancer cells, Global Journal of Cancer Therapy 6 (2020), 001-003.

\section{Figures}




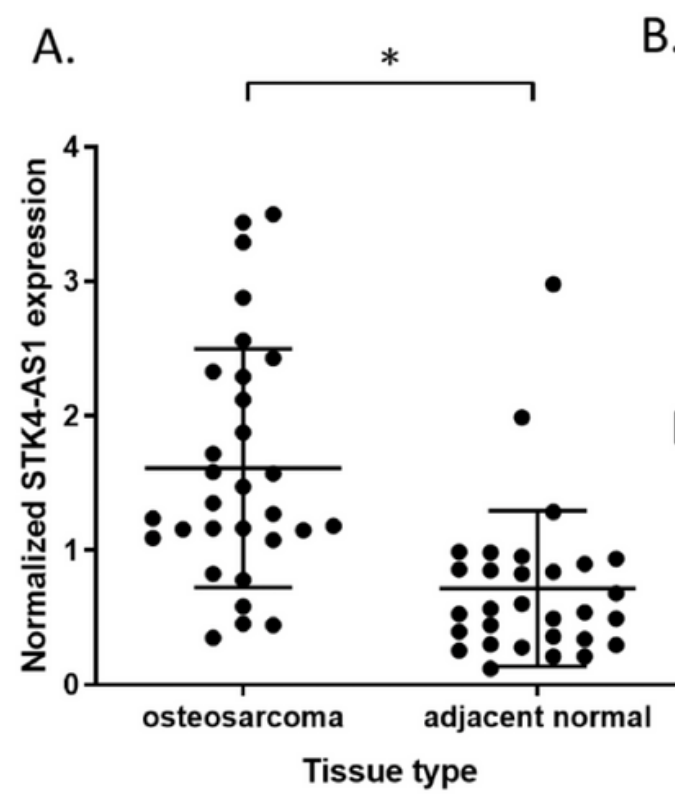

Tissue type
B.

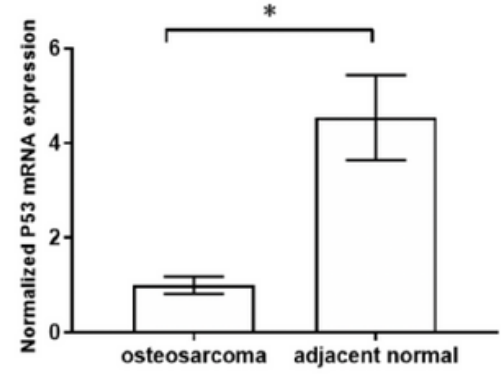

D.

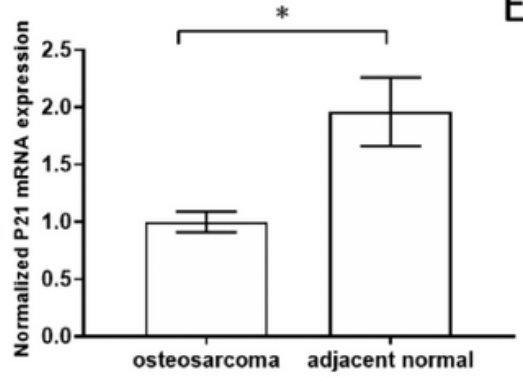

Tissue type
C.

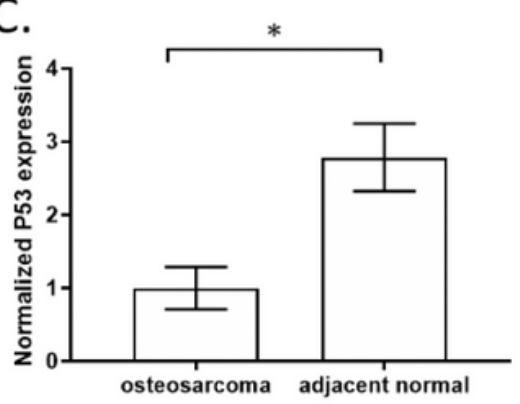

E.

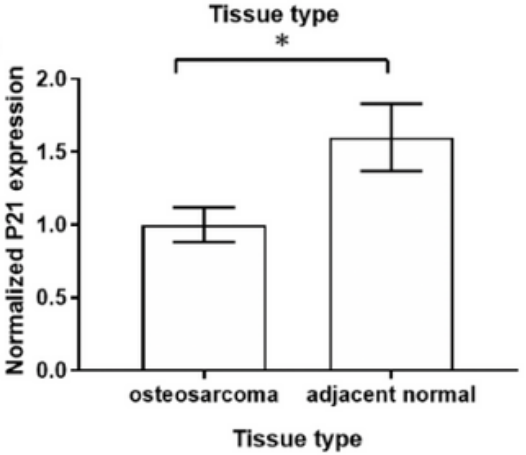

F.

P53

adjacent normal

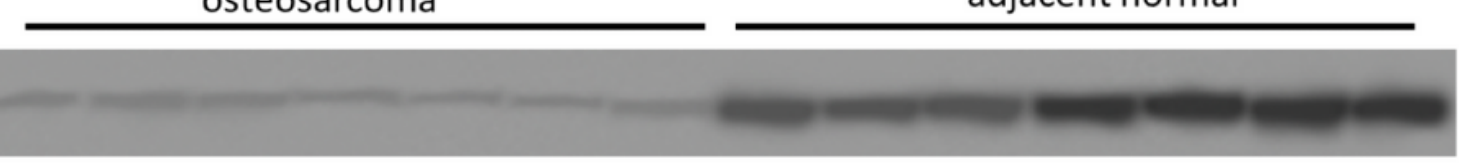

$50 \mathrm{kDa}$

P21

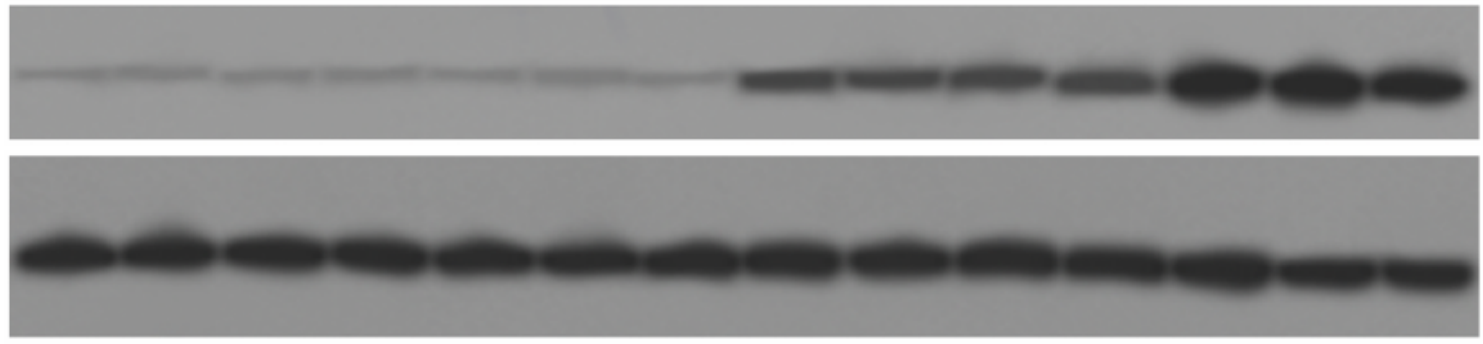

$20 \mathrm{kDa}$

GAPDH

Slope $=-0.09204$

G.

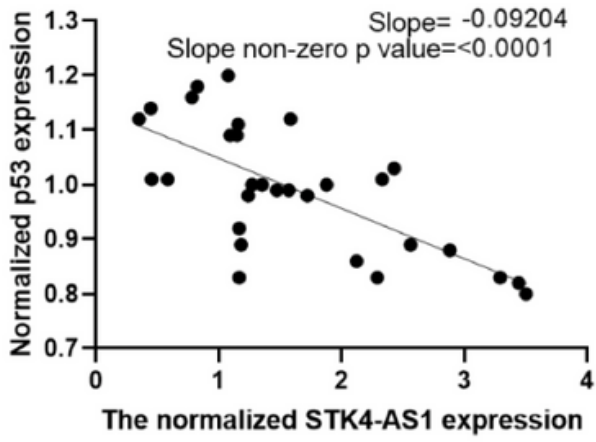

$\mathrm{H}$.

Slope $=-0.06830$

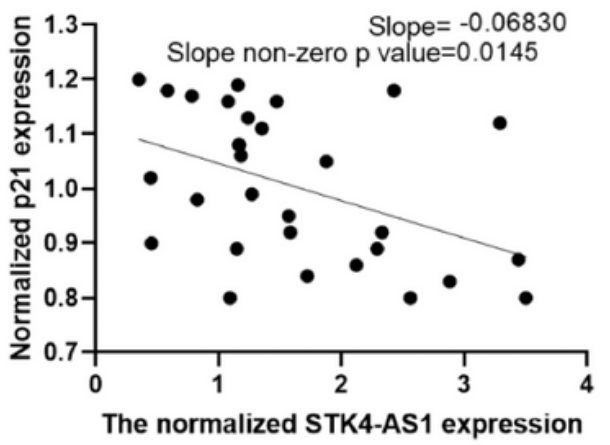

37 kDa

\section{Figure 1}

STK4-AS1, p53, and p21 expression in osteosarcoma and osteoblast. The STK4-AS1 and p53 and p21 mRNA expression were determined using QPCR assay. The p53 and p21 protein expressions were determined using western blotting. (A) STK4-AS1 expression in human osteosarcoma and adjacent normal tissues from 30 patients with osteosarcoma resection. (B) p53 mRNA expression in human osteosarcoma and adjacent normal tissues. (C) p53 protein expression in human osteosarcoma and adjacent normal tissues. (D) p21 mRNA expression in human osteosarcoma and adjacent normal 
tissues. (E) p21 protein expression in human osteosarcoma and adjacent normal tissues. (F) Representative images of western blotting assay of tissues. (G) Correlation between STK4-AS1 and p53 expression in osteosarcoma tissues. $(\mathrm{H})$ Correlation between STK4-AS1 and p21 expression in osteosarcoma tissues. $\left({ }^{*} p<0.01\right.$.)

A.

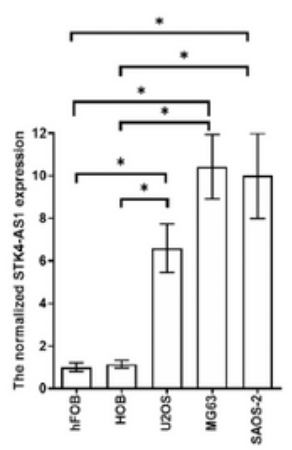

B.

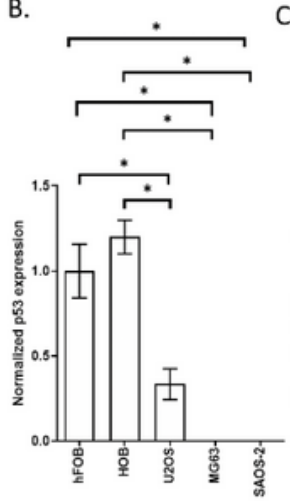

C.

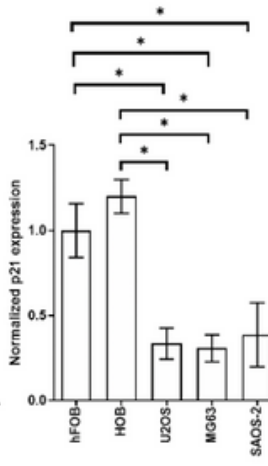

D.

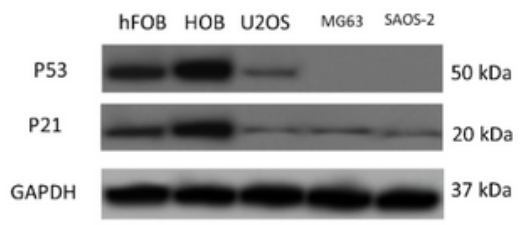

E.

U20S

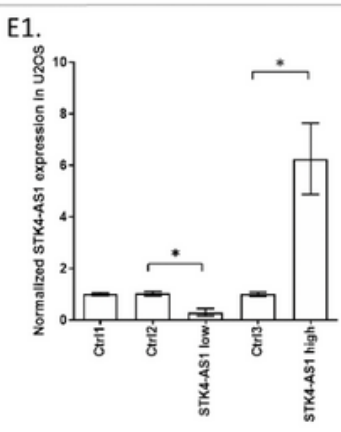

E2.

E3.

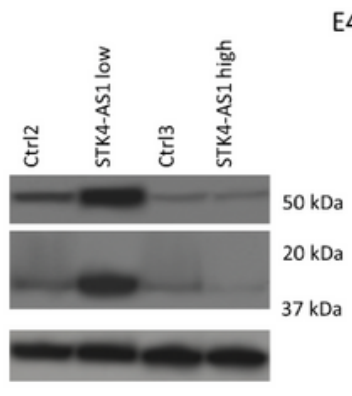

E4.

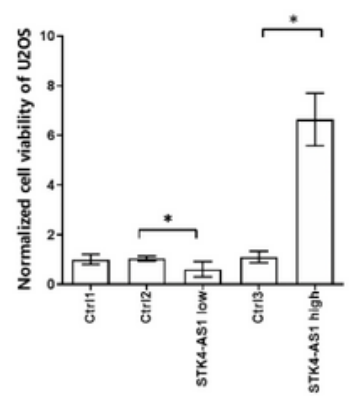

F.
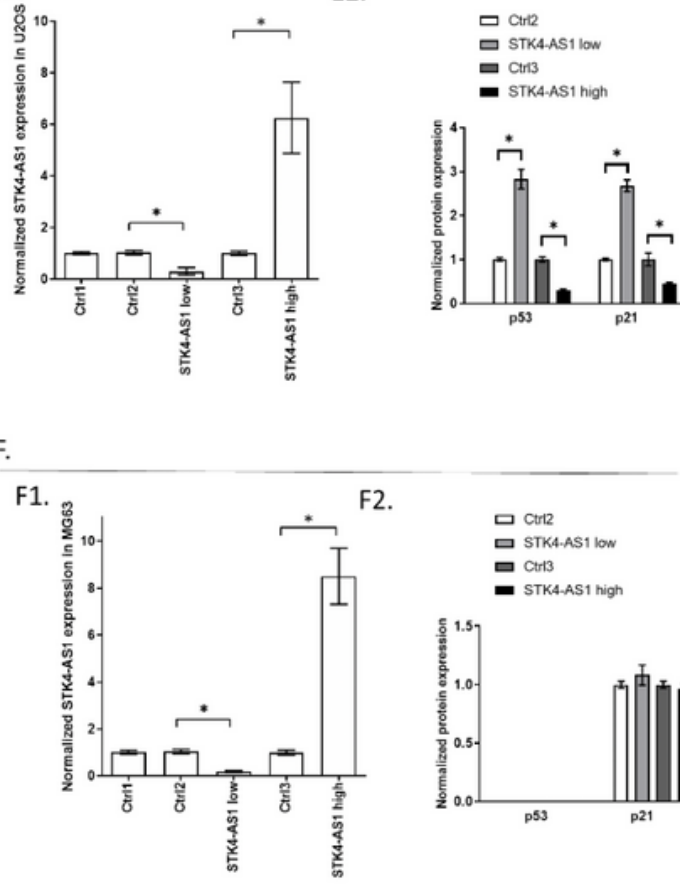

G.

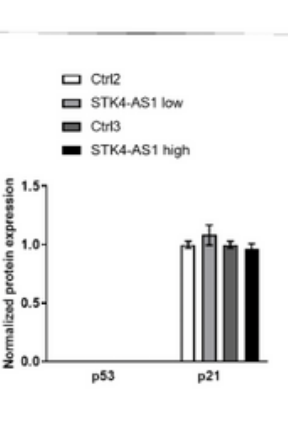

MG63

F3.

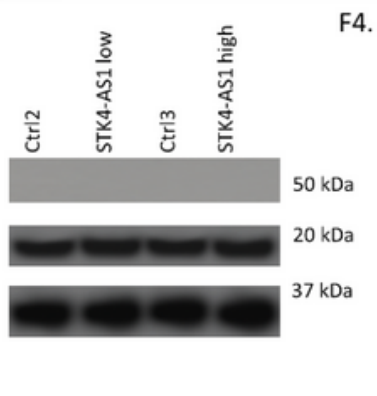

F4.

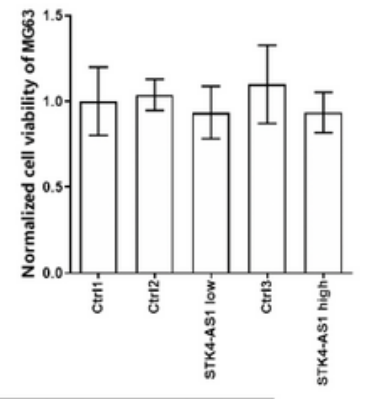

hFOB

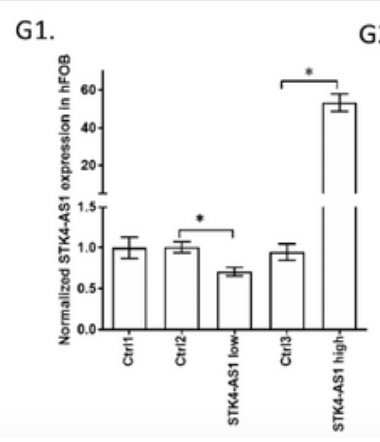

G2. 므남

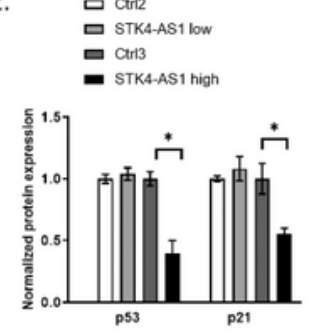

G3.

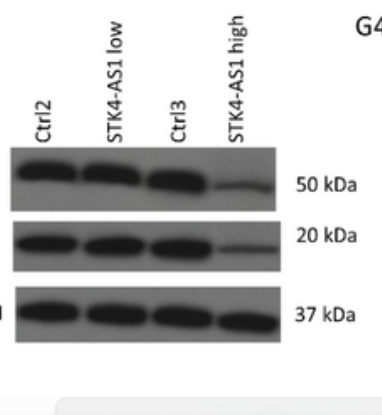

G4.

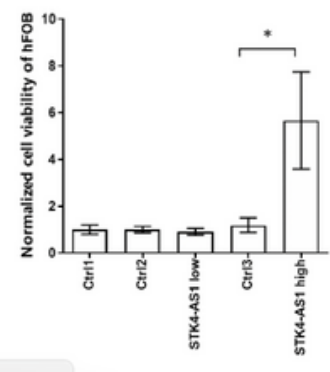

Figure 2 
STK4-AS1 in osteosarcoma and osteoblast cells. (A) STK4-AS1 expression in human osteosarcoma and osteoblast cells. (B) p53 protein expression in human osteosarcoma and osteoblast cells. (C) p21 protein expression in human osteosarcoma and osteoblast cells. (D) Representative images of western blotting assay of human osteosarcoma and osteoblast cells. The STK4-AS1 and mRNA expression were determined using QPCR and the protein expression was determined using western blotting. The cell viability was determined using the MTT assay. (E) Knockdown or overexpression of STK4-AS1 in osteosarcoma cells U2OS. E1. STK4-AS1 expression in U2OS. E2. Protein expression of p53 and p21 in U2OS. E3. Representative image of western blotting of p53 and p21 in U20S. E4. The cell viability of U2OS. (F) Knockdown or overexpression of STK4-AS1 in osteosarcoma cells MG63. F1. STK4-AS1 expression in MG63. F2. Protein expression of p53 and p21 in MG63. F3. Representative image of western blotting of p53 and p21 in MG63. F4. The cell viability of MG63. (G) Knockdown or overexpression of STK4-AS1 in osteoblast cells hFOB. G1. STK4-AS1 expression in hFOB. G2. Protein expression of p53 and p21 in hFOB. G3. Representative image of western blotting of p53 and p21 in hFOB. G4. The cell viability of hFOB. ( ${ }^{*} p<0.01$. Ctrl 1: cells without treatment; $c$ trl 2 : cells with negative control siRNA; ctrl 3: cells with negative control empty vector. ) 
A.

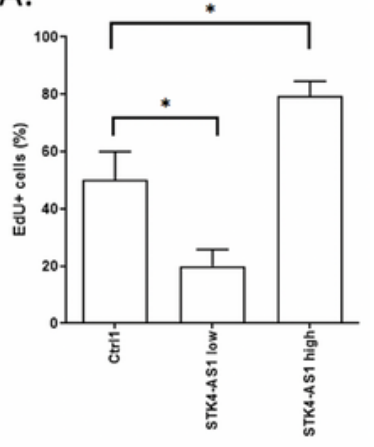

D.
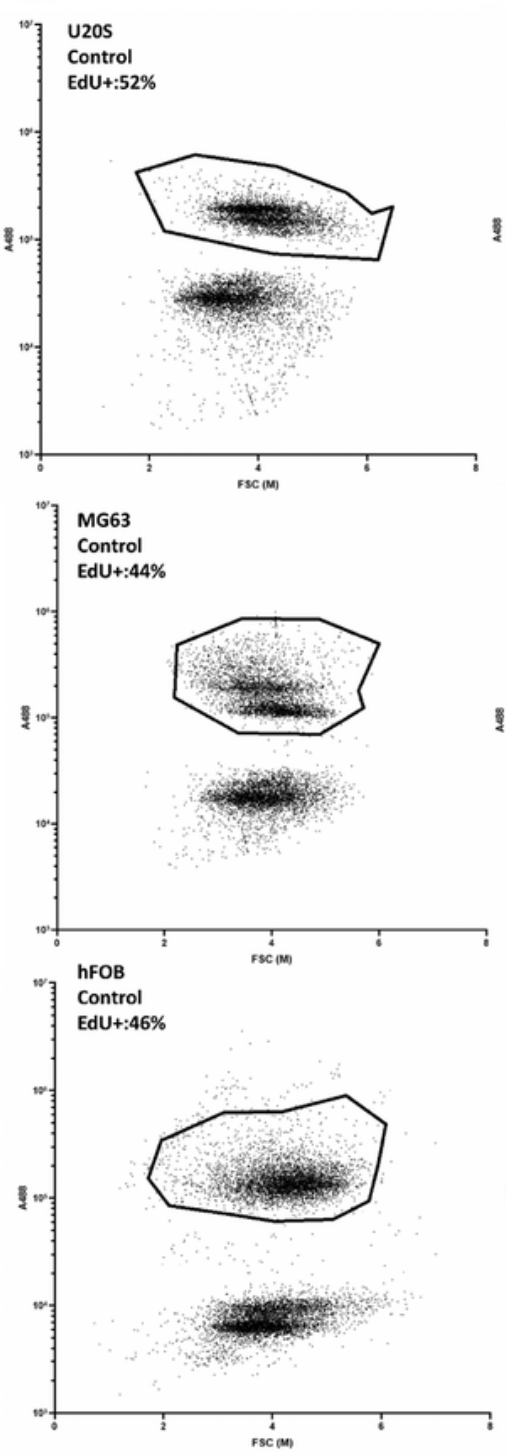

B.
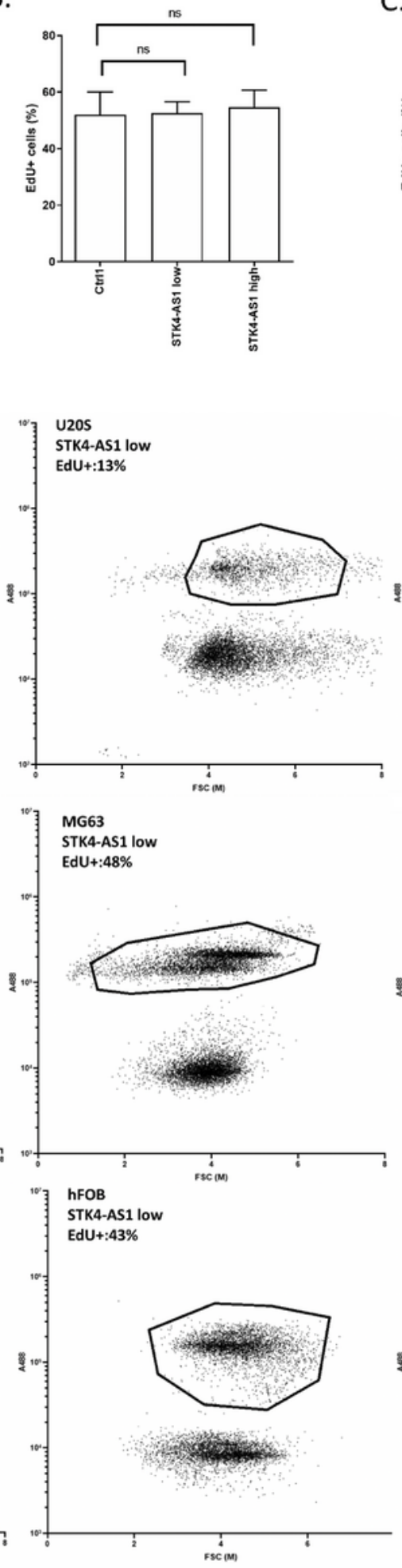

C.
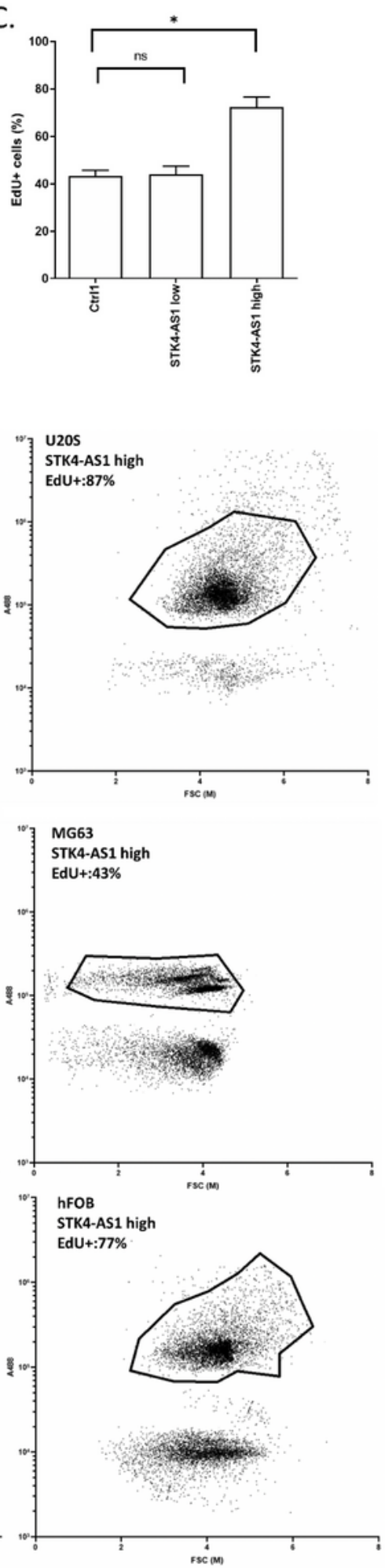

Figure 3

Effects of knockdown or overexpression of STK4-AS1 on cells proliferation. Cells proliferation levels were analyzed using EdU assay. (A) Effects of knockdown or overexpression of STK4-AS1 on U2OS cells proliferation. (B) Effects of knockdown or overexpression of STK4-AS1 on MG63 cells proliferation. (C) Effects of knockdown or overexpression of STK4-AS1 on hFOB cells proliferation. (D) Representative 
image of EdU cells in flow cytometry. ( ${ }^{\star} p<0.01$. Ctrl 1: cells without treatment; ctrl 2: cells with negative control siRNA; ctrl 3: cells with negative control empty vector. )

A.

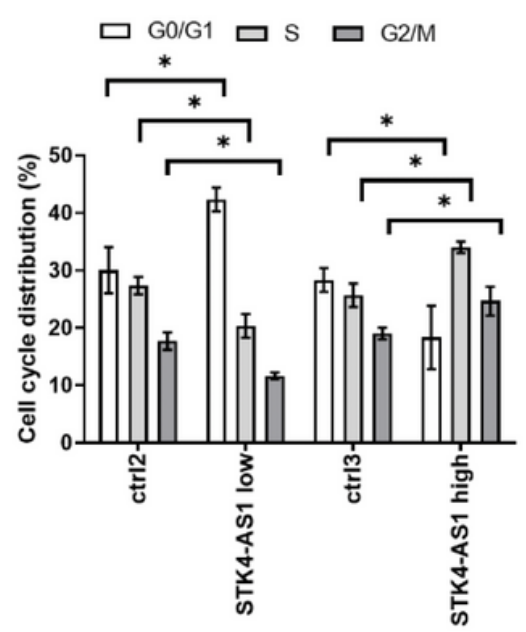

C.

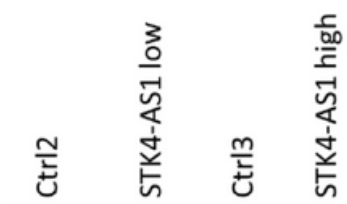

Cyclin A

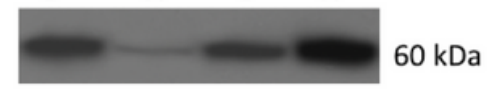

Cyclin B

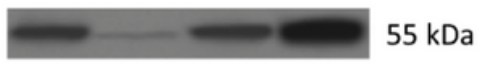

Cyclin D

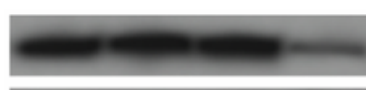

Cyclin $E$

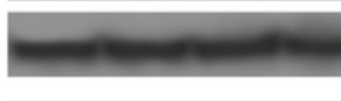

GAPDH
B.

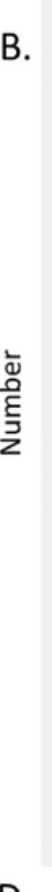

D.

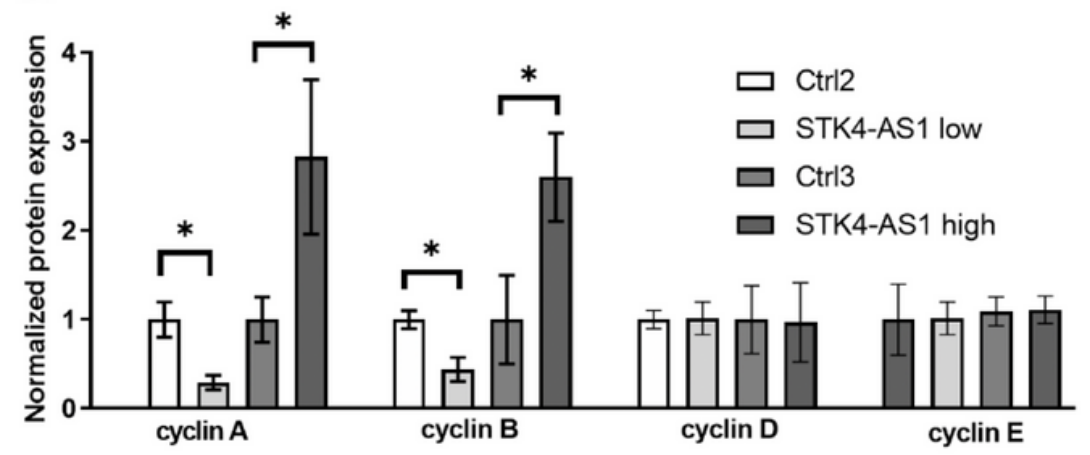

Figure 4

Effect of STK4-AS1 on the U2OS cell cycle. (A) Effect of knockdown and overexpression of STK4-AS1 on U2OS cell cycle. (B) Representative images of cell cycle analysis. (C) Representative images of western blotting for cyclin proteins. (D) Effect of knockdown and overexpression of STK4-AS1 on the level of cyclin proteins in U2OS. ( ${ }^{\star} p<0.01$. Ctrl 1: cells without treatment; ctrl 2: cells with negative control siRNA; ctrl 3: cells with negative control empty vector. ) 

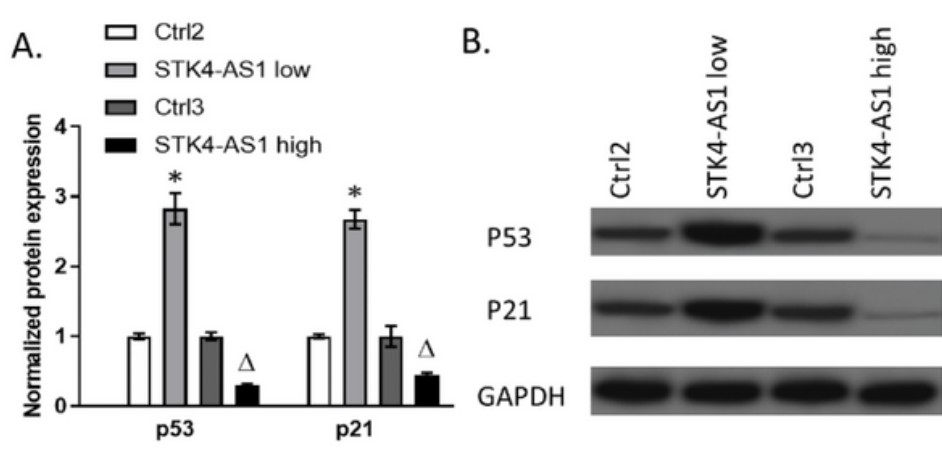

50 kDa

20 kDa

$37 \mathrm{kDa}$

C.

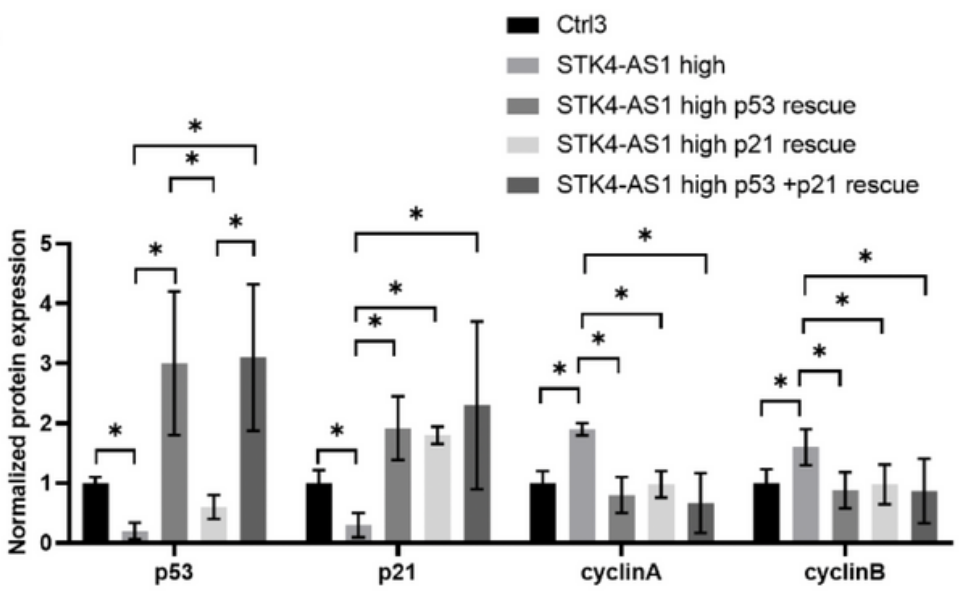

D.
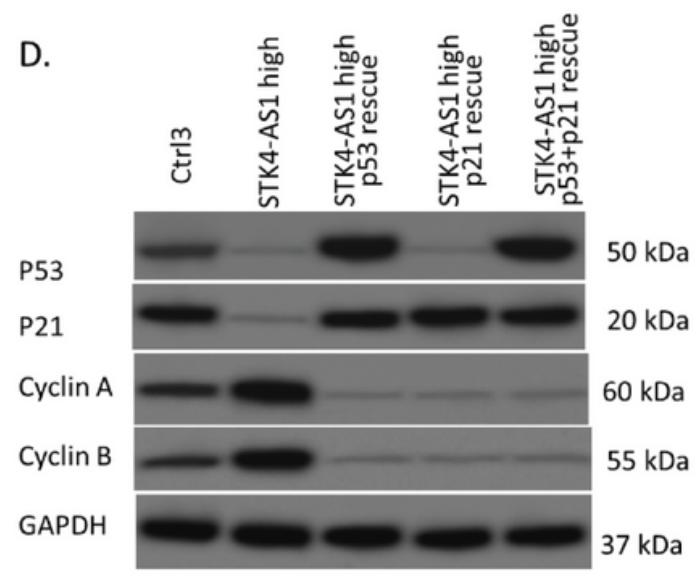

E.

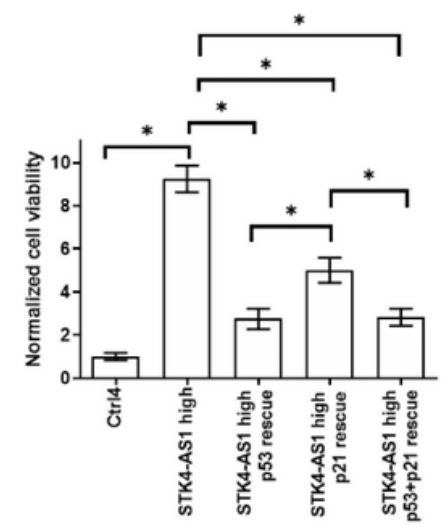

\section{Figure 5}

The effect of STK4-AS1 on p53/p21 regulation in U2OS cells. Protein expression was determined using western blotting and the cell viability was determined using the MTT assay. (A) Effect of STK4-AS1 on the expression of p53 and p21. (B) Representative images of p53/p21 western blotting in U2OS. (C) Protein expression of STK4-AS1 overexpressed U2OS cells with p53 or p21 rescue. (D) Representative images of p53/p21 western blotting in U2OS rescue experiments. (E) The cell viability of STK4-AS1 overexpressed U20S cells with $\mathrm{p} 53$ or $\mathrm{p} 21$ rescue. $\left({ }^{*} \mathrm{p}<0.01\right.$. Ctrl 1: cells without treatment; ctrl 2 : cells with negative control siRNA; ctrl 3: cells with negative control empty vector. ) 
A.

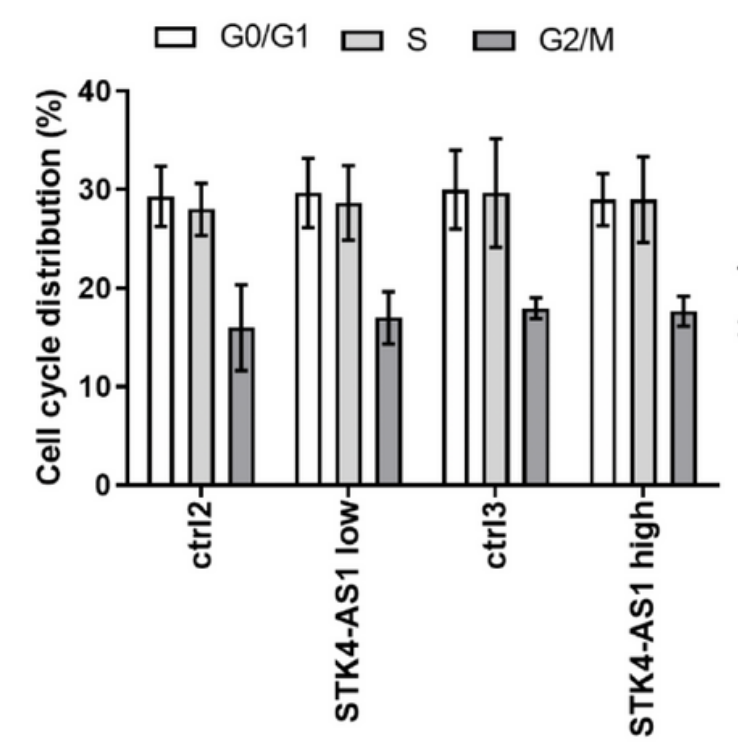

C.

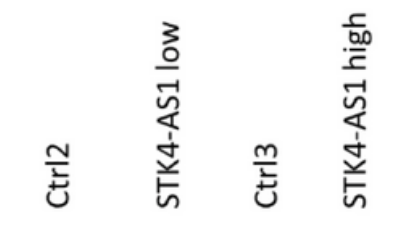

Cyclin A

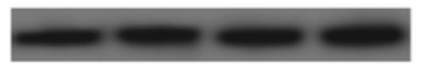

Cyclin B

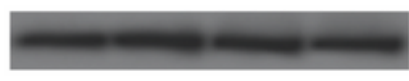

GAPDH

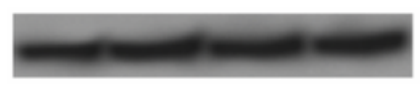

B.
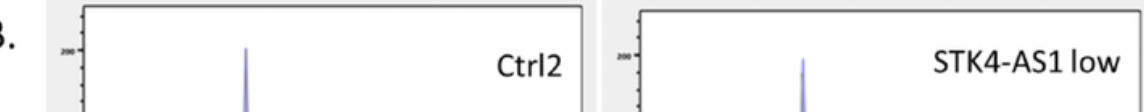

D.

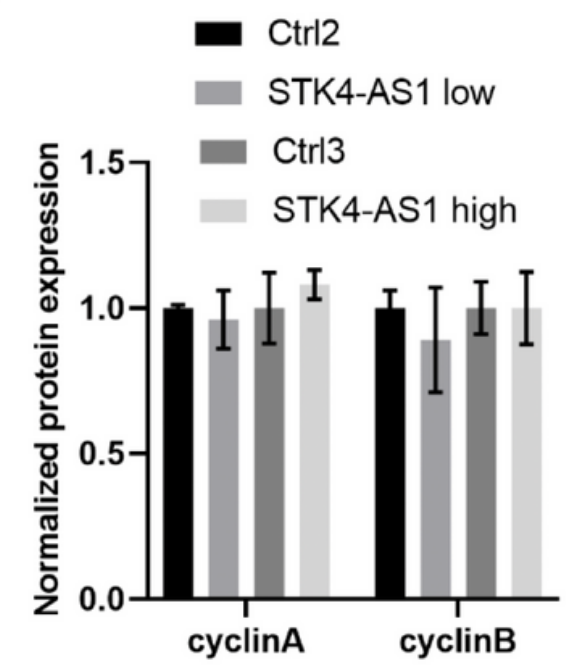

\section{Figure 6}

Effect of STK4-AS1 on the cell cycle of MG63 cells. (A) Effect of knockdown and overexpression of STK4AS1 on MG63 cell cycle. (B) Representative images of cell cycle analysis. (C) Protein expression of STK4AS1 overexpressed MG63 cells with p53 or p21 rescue. (D) Representative images of p53/p21 western blotting in MG63 rescue experiments. (E) The cell viability of STK4-AS1 overexpressed MG63 cells with p53 or p21 rescue. ( ${ }^{\star} p<0.01$. Ctrl 1: cells without treatment; ctrl 2: cells with negative control siRNA; ctrl 3: cells with negative control empty vector. ) 

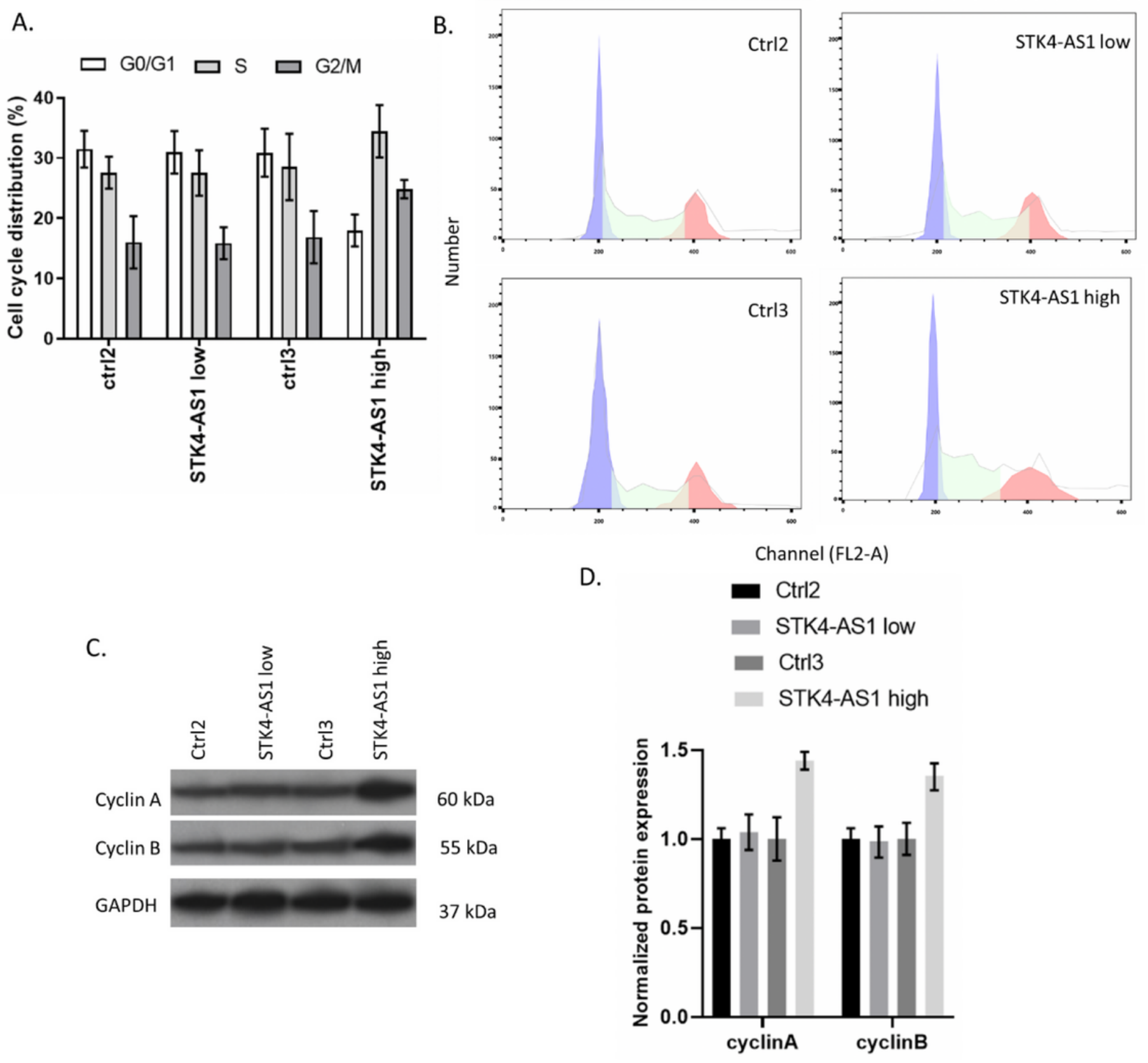

Figure 7

Effect of STK4-AS1 on the cell cycle of hFOB. (A) Effect of knockdown and overexpression of STK4-AS1 on hFOB cell cycle. (B) Representative images of cell cycle analysis. (C) Protein expression of STK4-AS1 overexpressed hFOB cells with p53 or p21 rescue. (D) Representative images of p53/p21 western blotting in hFOB rescue experiments. (E) The cell viability of STK4-AS1 overexpressed hFOB cells with p53 or p21 rescue. $\left({ }^{*} p<0.01\right.$. Ctrl 1 : cells without treatment; $c$ trl 2 : cells with negative control siRNA; ctrl 3 : cells with negative control empty vector. ) 

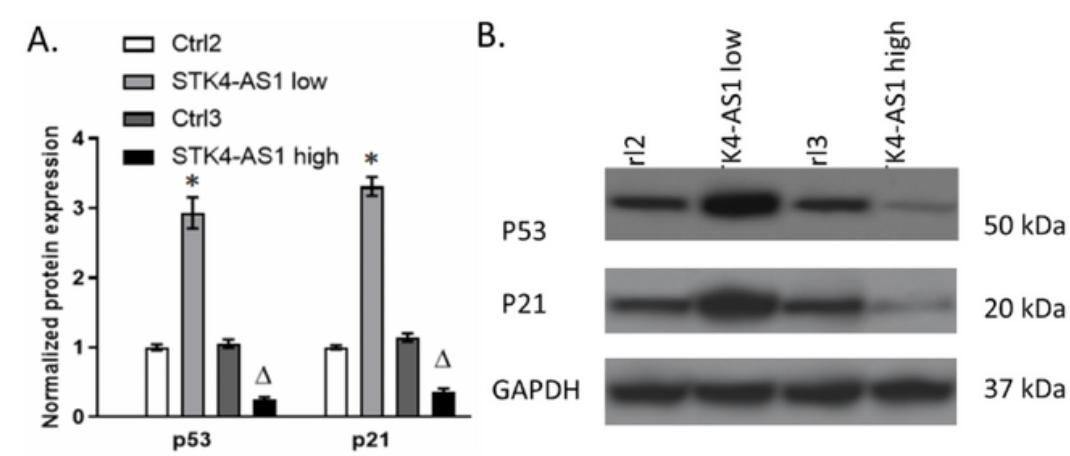

C.

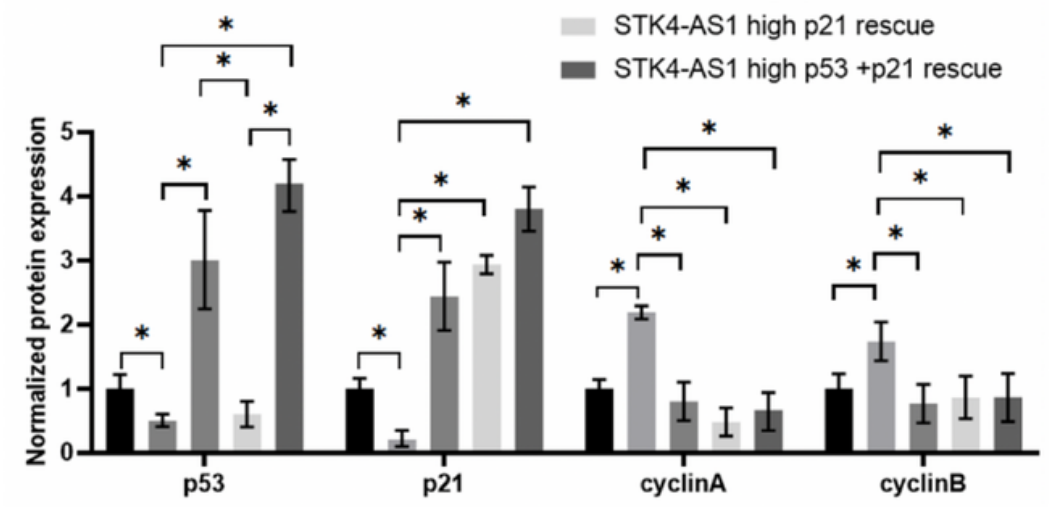

D.

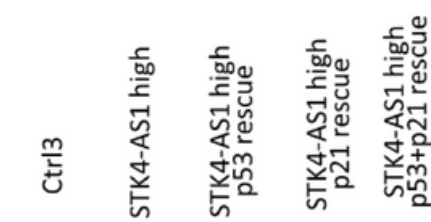

E.
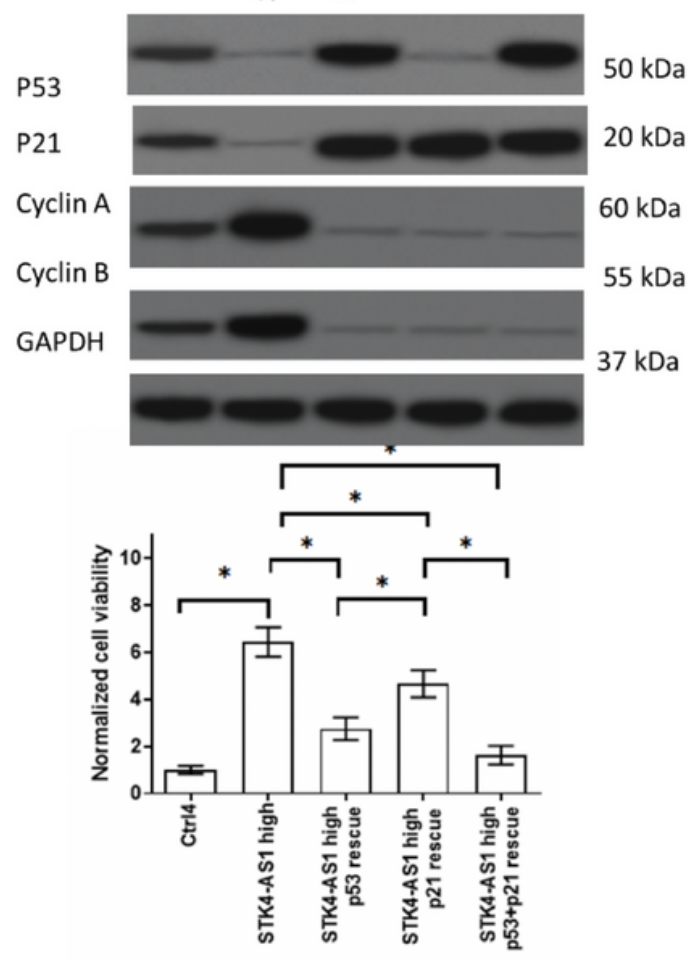

\section{Figure 8}

The effect of STK4-AS1 on p53/p21 regulation in hFOB cells. Protein expression was determined using western blotting and the cell viability was determined using the MTT assay. (A) Effect of STK4-AS1 on the expression of p53 and p21. (B) Representative images of p53/p21 western blotting in hFOB. (C) Protein expression of STK4-AS1 overexpressed hFOB cells with p53 or p21 rescue or p53/p21 co-rescue. (D) Representative images of p53/p21 western blotting in $\mathrm{hFOB}$ rescue experiments. (E) The cell viability of STK4-AS1 overexpressed hFOB cells with p53 or p21 rescue. ( ${ }^{*} p<0.01$. Ctrl 1: cells without treatment; ctrl 2: cells with negative control siRNA; ctrl 3: cells with negative control empty vector. )

\section{Figure 9}

Interaction of STK4-AS1 and TP53. The analysis was conducted using LncRRIsearch. (A) Subcellular localization (subcytoplasm and subnucleus compartments) of STK4-AS1 and TP53 mRNA. (B) Global base-pairing interaction analysis of STK4-AS1 and TP53. 


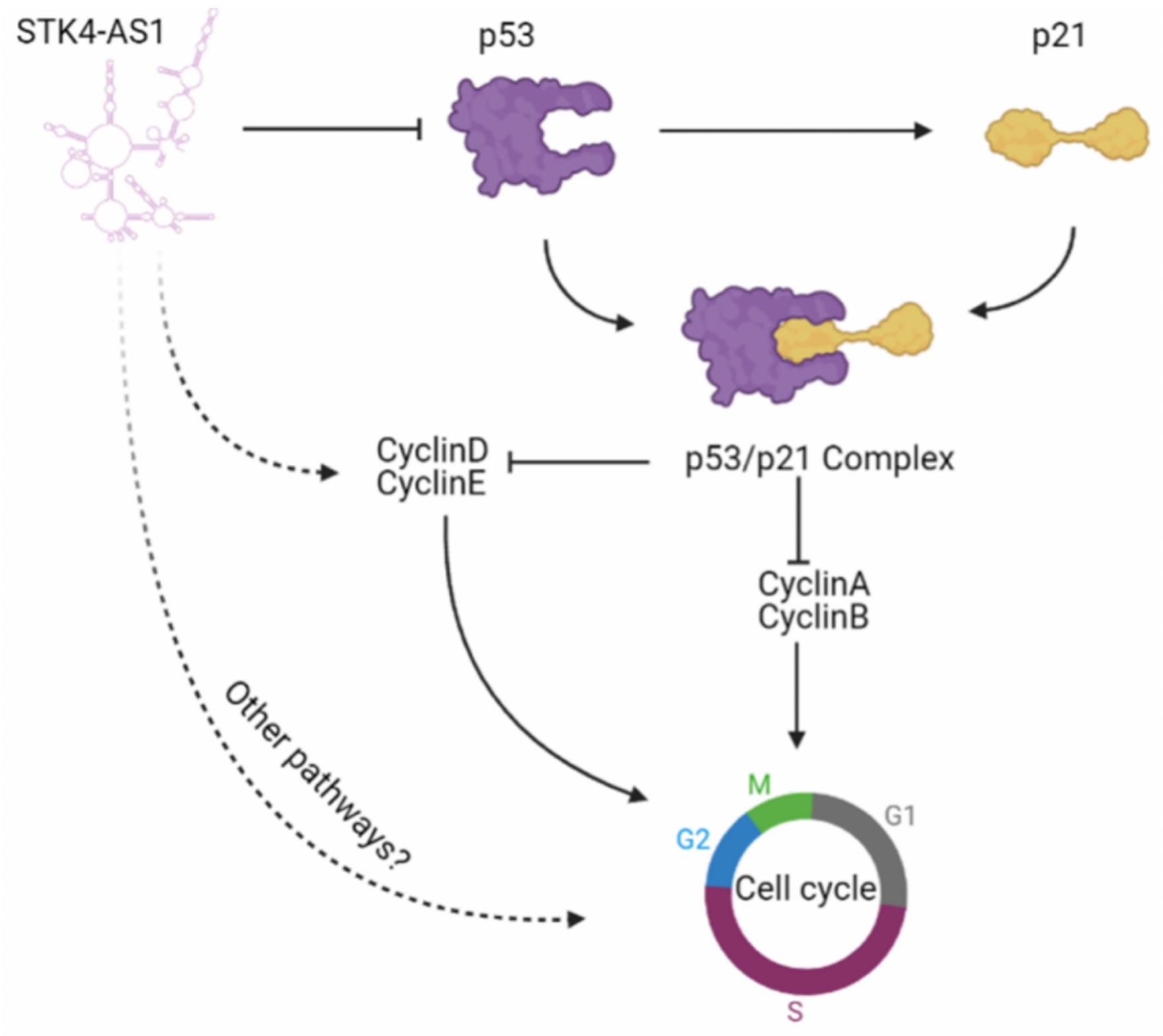

Figure 10

Proposed model for the role of STK4-AS1 in osteosarcoma cell cycle regulation.

\section{Supplementary Files}

This is a list of supplementary files associated with this preprint. Click to download.

- supplimentary.docx 